\title{
Erythrolamprus miliaris orinus (Reptilia, Squamata, Dipsadidae): tentativas de predação de Boana faber e Leptodactylus latrans
}

\section{Erythrolamprus miliaris orinus (Reptilia, Squamata, Dipsadidae): predation attempts on Boana faber and Leptodactylus latrans (Amphibia, Anura)}

\author{
Ana Beatriz Carollo Rocha-Lima', "II, Isaías Santos "III, Letícia Suigh Carlos Duarte", William Pinheiro da Costal \\ Universidade Estadual de Campinas. Campinas, São Paulo, Brasil \\ "Universidade Paulista. Jundiaí, São Paulo, Brasil \\ IIIReserva Betary. Centro de Estudos da Biodiversidade. Iporanga, São Paulo, Brasil
}

\begin{abstract}
Resumo: A presente comunicação relata duas tentativas de predação envolvendo subadultos de Erythrolamprus miliaris orinus e duas espécies de anfíbios anuros de grande porte, bem como evidencia as possíveis estratégias da serpente para vencer sua presa diante da dificuldade em deglutir o indivíduo. Em ambos os casos, os anfíbios obtiveram êxito e escaparam do predador. $O$ fato de os dois indivíduos apresentarem tamanho relativamente grande (entre 60 e $70 \mathrm{~mm}$ de comprimento rostro-cloacal) em relação à maioria dos anfíbios possivelmente favoreceu a fuga dos indivíduos, bem como a desistência da serpente em efetivar a predação. As estratégias aqui relatadas, em alguns casos, podem aumentar a eficiência da predação quando a presa for relativamente grande ou apresentar resistência. $O$ tipo de substrato, a relação de tamanho entre presa e predador, bem como a experiência em forrageio da serpente podem também ser fatores limitantes para determinar a eficiência na captura.
\end{abstract}

Palavras-chave: Dieta. Comportamento alimentar. Anura. Forrageamento. Subjugação.

Abstract: This communication reports two predation attempts involving subadults of the snake Erythrolamprus miliaris orinus on two species of large anurans, as well as the possible strategies of the snake in the face of difficulty to ingest the prey individual. In both cases, amphibians were successful in escaping the predator. The fact that the two individuals were relatively large (between 60 and $70 \mathrm{~mm}$ snout-vent length) compared to most amphibians possibly favored the escape, as well as the withdrawal of the snake in effecting the predation. The strategies reported here, in some cases, can increase predation efficiency when the prey is relatively large or resistant. The type of substrate, the size relation between prey and predator and the experience of the snake also can be limiting factors to determine the efficiency of capture.

Keywords: Diet. Feeding behavior. Anura. Foraging. Subjugation.

ROCHA-LIMA, A. B. C., I. SANTOS, L. S. C. DUARTE \&W. P. COSTA, 2018. Erythrolamprus miliaris orinus (Reptilia, Squamata, Dipsadidae): tentativas de predação de Boana faber e Leptodactylus latrans. Boletim do Museu Paraense Emílio Goeldi. Ciências Naturais 13(3): $455-460$. Autora para correspondência: Ana Beatriz Carollo Rocha-Lima. Universidade Paulista. Campus Jundiaí. Instituto de Ciências da Saúde. Av. Armando Giassetti, 577 - Trevo Itu/Itatiba - Vila Hortolândia. Jundiaí, SP, Brasil. CEP 13214-525 (abeatrizcrl@gmail.com).

Recebido em 09/01/2018

Aprovado em 25/05/2018

Responsabilidade editorial: Fernando da Silva Carvalho Filho

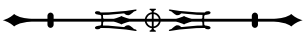




\section{INTRODUÇÃO}

A dieta é um dos principais aspectos associados ao nicho ecológico das serpentes, tendo influência sobre o comportamento alimentar, o habitat e o período de atividade dessas espécies (Toft, 1985). Entre as serpentes existentes na América do Sul, a família Dipsadidae (que inclui o gênero Erythrolamprus) possui grande número de representantes adaptados à predação de anfíbios, com diversas espécies anurófagas (Michaud \& Dixon, 1989; Martins et al., 1993; Bernarde \& Abe, 2010). Erythrolamprus miliaris (Linnaeus, 1758) apresenta hábito semiaquático, sendo frequentemente encontrada próxima a corpos d'água utilizados por diferentes grupos de anuros durante o período reprodutivo (Sazima \& Haddad, 1992; Marques \& Sazima, 2004). Além disso, essa é uma espécie politípica que inclui as subespécies E. miliaris miliaris (Linnaeus, 1758), E. m. amazonicus (Dunn, 1922), E. m. chrysostomus (Cope, 1868), E. m. merremii (Wied, 1821) e E. m. orinus (Griffin, 1916), sendo esta última a subespécie que ocorre em São Paulo, no sul de Minas Gerais, no Rio de Janeiro, no Paraná, em Santa Catarina e no Rio Grande do Sul (Lima, A. C., 2012), no Brasil, bem como nos chacos úmidos da Argentina e do Paraguai (Giraudo et al., 2006).

O comportamento gregário, característico de muitos anfíbios, torna-os presas em potencial para todas as classes de vertebrados (Duellman \& Trueb, 1986), incluindo as serpentes. Ainda que uma das principais causas da redução do número efetivo de indivíduos em uma comunidade de anfíbios seja a pressão de predação (Duellman \& Trueb, 1986), e que eventos dessa natureza envolvendo serpentes e anfíbios estejam sendo documentados (Martins et al., 1993; Bovo \& Sueiro, 2012; Forti \& Bertoluci, 2012; Moya \& Maffei, 2012), a eficiência da captura de anuros adultos por serpentes é pouco abordada.

Neste trabalho, são relatadas duas tentativas de predação sem êxito envolvendo subadultos de E. m. orinus e dois indivíduos de anfíbios anuros considerados de grande porte, apresentando entre 60 e $70 \mathrm{~mm}$ de comprimento rostro-cloacal (CRC), Boana faber (Wied-Neuwied, 1821) e Leptodactylus latrans (Steffen, 1815). Foram apresentadas também as possíveis estratégias da serpente para subjugar sua presa diante da dificuldade em deglutir o indivíduo, em função de este ser de tamanho relativamente grande.

\section{MATERIAL E MÉTODOS}

As observações relatadas basearam-se em encontros ocasionais de eventos predatórios em duas áreas distintas. A observação foi realizada a uma distância suficientemente necessária para não haver interferência no comportamento dos indivíduos.

$\mathrm{O}$ primeiro evento foi registrado entre 18:20 $\mathrm{h}$ e 19:21 h do dia 3 de outubro de 2010, no interior de um pneu, próximo a uma poça temporária em uma área aberta, com predomínio de vegetação de gramíneas ( $24^{\circ} 35^{\prime} 00^{\prime \prime} \mathrm{S}$ e $48^{\circ} 35^{\prime} 33^{\prime \prime} \mathrm{O}$ ), no município de Iporanga, estado de São Paulo, Brasil.

O segundo evento foi registrado entre 19:45 h e 22:25 h do dia 17 de setembro de 2012, em uma lagoa (23 23'46" S e 46 93'36" O), na serra do Japi, no município de Jundiaí, estado de São Paulo, Brasil.

\section{RESULTADOS}

De acordo com a observação e a análise feita das tentativas de predação, três estratégias para minar as forças do anuro puderam ser identificadas: (i) puxar o indivíduo para o fundo do corpo d'água (Figura 1); (ii) virar o indivíduo com o ventre para cima em movimentos circulatórios (Figura 2); e (iii) impulsionar o anuro contra o substrato (Figura 3).

O primeiro evento observado refere-se à tentativa de predação de um indivíduo adulto de $L$. latrans por um subadulto de $E$. m. orinus de aproximadamente $60 \mathrm{~cm}$ de comprimento. $\bigcirc$ evento foi detectado após ser percebido o grito de agonia emitido pela rã, proveniente do interior do pneu. Ao se aproximar do local, visualizou-se que a serpente, em um momento de oportunismo, deu o bote em sua presa, apreendendo a perna esquerda da rã na posição dorsal (Figura 4). Após sua captura, a presa

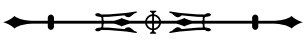




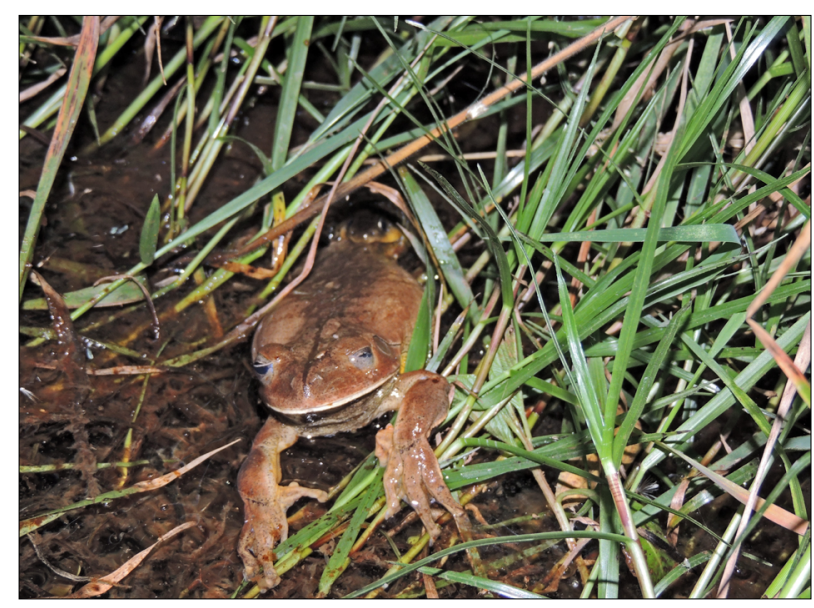

Figura 1. E. m. orinus tentando puxar B. faber para dentro da água (estratégia i). Foto: W. P. da Costa (2012).

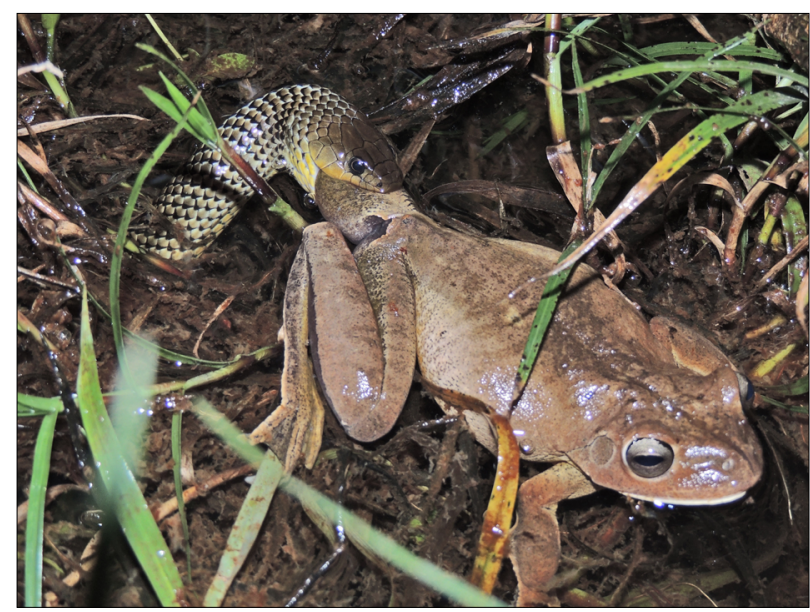

Figura 3. E. m. orinus investindo contra B. faber parcialmente fora da água (estratégia iii). Foto: W. P. da Costa (2012).

ofereceu forte resistência, segurando-se na vegetação contida no interior do pneu. Por sua vez, a serpente realizou constantes movimentos circulatórios, na tentativa de minar a resistência da presa (estratégia ii), entretanto, o anuro acompanhou o movimento da serpente girando no mesmo sentido, evitando o possível deslocamento de seu membro posterior. Em momentos de menor agitação da serpente, a rã realizou movimentos repentinos, na tentativa de liberar sua perna da boca da serpente.

O embate durou mais de 30 minutos, até que a serpente desistiu da presa, afastando-se rapidamente do

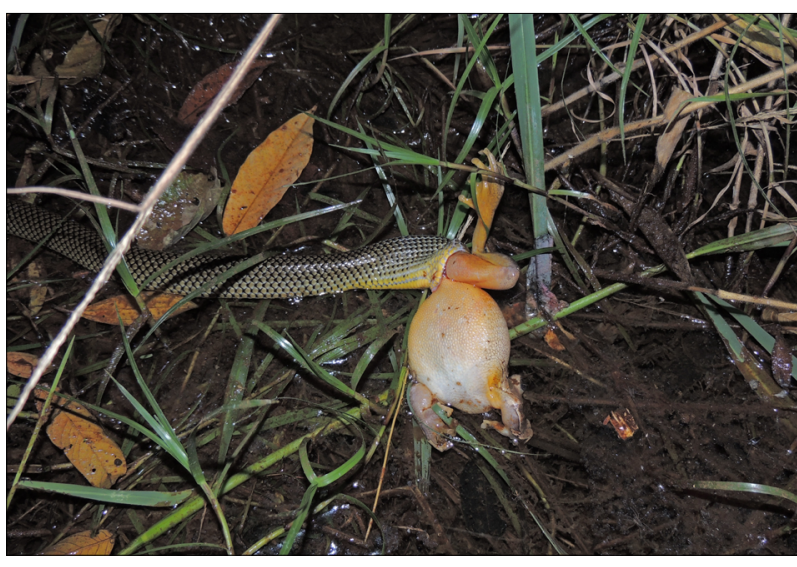

Figura 2. E. m. orinus, em movimentos circulatórios, tentando virar B. faber com o ventre para cima (estratégia ii). Foto: W. P. da Costa (2012).

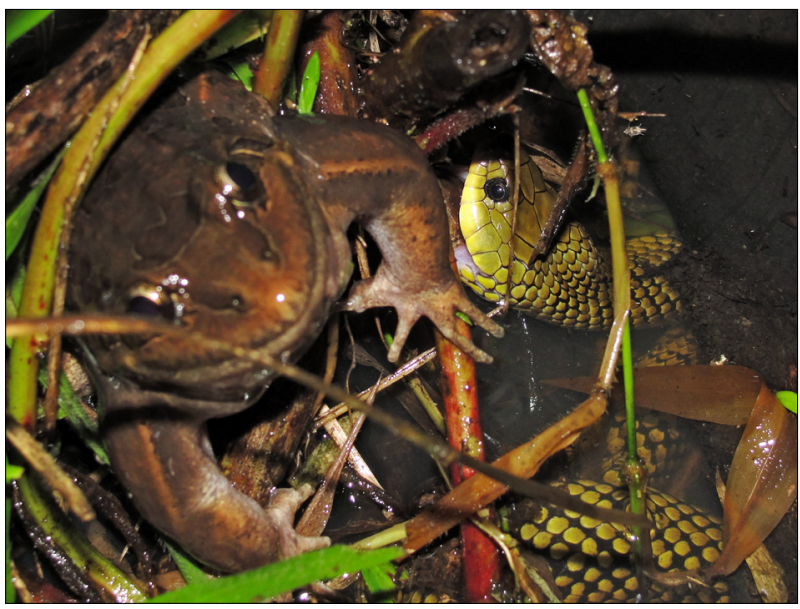

Figura 4. E. m. orinus apreendendo a pata traseira de L. latrans (estratégia ii). Foto: I. Santos (2010).

local. A rã, vendo-se livre, também saiu velozmente do interior do pneu, deslocando-se sobre a vegetação, sendo, então, capturada para o estudo. $\bigcirc$ voucher encontra-se tombado na coleção de anfíbios do Museu de Zoologia da Universidade de Campinas (ZUEC), sob o tombo de número 16838.

O segundo evento observado refere-se à tentativa de predação de um indivíduo adulto de $B$. faber por um subadulto de $E$. m. orinus de aproximadamente $70 \mathrm{~cm}$ de comprimento. Foram identificados cinco indivíduos de $B$. faber em atividade normal de vocalização sobre vegetação

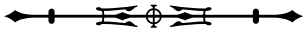


arbóreo-arbustiva nas margens do corpo d'água, e apenas um indivíduo vocalizando em vegetação rente ao lago. Na sequência, ouviu-se um intenso grito de agonia vindo de um ponto a cerca de $3 \mathrm{~m}$ da margem, em uma área com predomínio de gramíneas emergentes, no interior do corpo d'água. Neste local, visualizou-se um macho de $B$. faber agarrado à vegetação emergente, alternando entre movimentos de tentativa de fuga e gritos de agonia, lutando contra a tentativa de predação de uma cobra d'água ( $E$. m. orinus).

A serpente também havia apreendido a presa pela perna esquerda, na posição dorsal, e o membro encontrava-se parcialmente deglutido. A serpente tentou sucessivas vezes puxar o indivíduo para o fundo do corpo d'água (estratégia i), porém o anuro se agarrou fortemente ao substrato e conseguiu resistir às tentativas. A serpente também tentou virar o indivíduo com o ventre para cima em movimentos circulatórios (estratégia ii), entretanto, assim como no evento anterior, o anuro acompanhou o movimento da serpente, girando no mesmo sentido, evitando, assim, o possível deslocamento de seu membro deglutido. Também foram observadas diversas investidas contra a presa em que a serpente saía com mais de $15 \mathrm{~cm}$ do corpo para fora da água, impulsionando o anuro contra o substrato, no qual este estava agarrado (estratégia iii).

O embate continuou por aproximadamente 40 minutos, sempre próximo à margem do lago. A evolução na tentativa de deglutição da serpente cessou quando ela atingiu a região inguinal, dado que a presa apresentou comportamento de inflar os pulmões e manteve a perna direita estendida para trás, aumentando, assim, a resistência contra a deglutição. Por fim, a serpente saiu de dentro do corpo d'água com a presa, regurgitando o membro esquerdo do anfíbio, com movimentos laterais do corpo em S, abandonando a tentativa de predação na margem da lagoa. A serpente refugiou-se no folhiço, desaparecendo rapidamente, e o anfíbio afastou-se com dificuldade do local onde foi solto pela serpente. Logo após a soltura, o indivíduo foi recolhido e verificamos um corte profundo no flanco direito, próximo à região inguinal, bem como marcas de dentes na perna esquerda, a qual manteve-se esticada durante todo o período em que o indivíduo foi manipulado, aparentando dificuldade de locomoção. $\bigcirc$ espécime foi levado ao laboratório, permanecendo vivo por mais duas horas. Após esse período, constatou-se a morte do indivíduo provavelmente em virtude da exaustão e perda de sangue. $O$ voucher encontra-se tombado na coleção de anfíbios do Museu de Zoologia da Universidade de Campinas (ZUEC), sob o tombo de número 19961.

\section{DISCUSSÃO}

São diversos os estudos que reportam Erythrolamprus miliaris (=Liophis miliaris) predando anuros grandes (gêneros Leptodactylus, Lithobates e Rhinella, Michaud \& Dixon, 1989; Leptodactylus labyrinthicus (Spix, 1824), Sazima \& Martins, 1990; Hylodes meridionalis (Mertens, 1927), Lima, A. F. \& Colombo, 2008). Estudos recentes têm desvendado o status taxonômico de E. miliaris (Giraudo et al., 2006; Lima, A. C., 2012); hoje, sabe-se que se trata de uma espécie politípica. Como não há dados na literatura que confirmem que todas as subespécies apresentam as mesmas estratégias alimentares, futuros estudos devem investigar a dieta e as diferentes estratégias alimentares para as subespécies de E. miliaris, já que estes aspectos da história natural podem variar entre as espécies e, até mesmo, entre os biomas onde elas ocorrem.

Boana faber, um anuro de grande porte, é uma presa comum entre as serpentes (Helicops modestus Günther, 1861, Martins et al., 1993; Thamnodynastes strigatus (Günther, 1858), Toledo et al., 2007), entre outros vertebrados (mamífero Cerdocyon thous (Linnaeus, 1766) e ave Tyto alba (Scopoli, 1769), Martins et al., 1993; anfibios Leptodactylus labyrinthicus e L. ocellatus (Linnaeus, 1758), Toledo et al., 2007) e até entre alguns invertebrados (larvas de libélula e baratas-d'água, Martins et al., 1993). Já os relatos que envolvem a predação de indivíduos adultos de $L$. latrans são mais escassos (barata-d'água Belostoma annulipes (Herrich-Schäffer, 1846), Lima, A. C., 1940; caranguejo

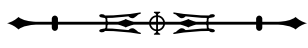


Dilocarcinus pagei Stimpson, 1861, Affonso \& Signorelli, 2011). Uma aparente vantagem em se alimentar de indivíduos relativamente grandes seria a elevada quantidade de energia obtida de uma só vez, porém um erro na avaliação pode ocasionar gasto de energia desnecessário, resultando em baixa eficiência na captura (Sazima \& Martins, 1990).

A grande maioria dos relatos de predação reporta a observação de eventos predatórios bem sucedidos e/ou baseiam-se na análise do conteúdo estomacal dos predadores (Michaud \& Dixon, 1989; Martins et al., 1993; Toledo et al., 2007; Lima, A. F. \& Colombo, 2008; Affonso \& Signorelli, 2011), enquanto os relatos de tentativas mal sucedidas são escassos (Sazima \& Martins, 1990; Forti \& Bertoluci, 2012), sendo praticamente inexistente a literatura sobre a análise da eficiência da captura e da taxa de sucesso de eventos predatórios. Sazima \& Martins (1990) consideram que tentativas de predar indivíduos de tamanho relativamente grande sejam um evento frequentemente associado a indivíduos jovens, que, por inexperiência, podem cometer um erro na avaliação do tamanho da presa.

Em relação às estratégias de resistência à predação pelos anuros, Forti \& Bertoluci (2012) reportaram um macho de $B$. faber emitindo gritos de agonia e tentando escapar para a frente, segurando a vegetação da grama com as mãos, enquanto a serpente a puxava para trás. Ambos os anuros incluídos na presente comunicação demonstraram a mesma estratégia. Assim como no evento reportado por Forti \& Bertoluci (2012), os indivíduos observados nesta pesquisa obtiveram êxito em escapar do predador. As estratégias de resistência à predação desenvolvidas pelos anuros possivelmente devem ter relevância para a sobrevivência de muitos indivíduos nos casos em que os predadores não subjugam a presa por inoculação de veneno.

Em ambos os relatos da presente comunicação, os anfíbios obtiveram êxito em escapar do predador, entretanto, no caso de B. faber, o indivíduo não sobreviveu. Segundo Martins et al. (1993), um dos principais fatores para a mortalidade de indivíduos de $B$. faber em uma comunidade é a pressão de predação. Aqui foi relatada a morte do indivíduo, não pela predação em si, mas sim por consequência à tentativa de predação de um indivíduo de $E$. $m$. orinus. Portanto, este aspecto aumenta a importância de E. m. orinus como fator de controle do efetivo de indivíduos da espécie em uma comunidade.

A complexidade taxonômica do gênero - ainda não completamente desvendada - aliada à falta de uma hipótese filogenética que suporte o monofiletismo do grupo são, no entanto, fatores que podem influenciar a existência de diferentes estratégias alimentares. Não existe, até o momento, nenhuma hipótese filogenética que comprove o monofiletismo deste grupo e, se for comprovado seu parafiletismo, isso pode refletir na variação das estratégias alimentares dos táxons em questão.

\section{CONCLUSÃO}

As estratégias de resistência aqui reportadas para as presas, aliadas ao fato de ambos os indivíduos apresentarem tamanho relativamente grande em relação à maioria dos anfíbios, possivelmente favoreceram a fuga dos indivíduos e ocasionaram a desistência da serpente em efetivar a predação.

As estratégias de subjugação das serpentes, em alguns casos, podem aumentar a eficiência da predação quando a presa for relativamente grande ou apresentar resistência. Ainda assim, o tipo de substrato, a relação de tamanho entre presa e predador e a experiência da serpente podem ser fatores limitantes para determinar a eficiência na captura, bem como o êxito dos anuros em escaparem do predador.

A presente comunicação pode contribuir para o entendimento do processo de predação realizado por serpentes e, mais especificamente, para elucidar aspectos do comportamento alimentar da subespécie $E$. m. orinus. Além disso, acrescenta novos registros de tentativas de predação de presas maiores do que os seus predadores, adicionando informações inéditas sobre os predadores de L. latrans.

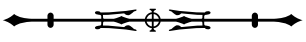




\section{AGRADECIMENTOS}

A L. Maretti e R. Pereira, da Secretaria do Meio Ambiente de Jundiaí, pelas autorizações concedidas. Aos professores L. F. Toledo e I. Sazima, pelas valiosas contribuições, e ao colega I. S. Oliveira, pela revisão do manuscrito.

\section{REFERÊNCIAS}

AFFONSO, I. P. \& L. SIGNORELLI, 2011. Predation on frogs by the introduced crab Dilocarcinus pagei Stimpson, 1861 (Decapoda, Trichodactylidae) on a neotropical floodplain. Crustaceana 84(12-13): 1653-1657.

BERNARDE, P. S. \& A. S. ABE, 2010. Hábitos alimentares de serpentes em Espigão do Oeste, Rondônia, Brasil. Biota Neotropica 10(1): 167-173. DOI: <http://dx.doi.org/10.1590/ S1676-06032010000100017>

BOVO, R. P. \& L. R. SUEIRO, 2012. Records of predation on Itapotihyla langsdorffii (Anura: Hylidae) by Chironius bicarinatus (Serpentes: Colubridae) with notes on foraging substrate. Herpetology Notes 5: 291-292.

DUELLMAN, W. E. \& L. TRUEB, 1986. Biology of amphibians: 1-670. Mc. Graw-Hill, New York.

FORTI, L. R. \&J. BERTOLUCI, 2012. Distress call of Hypsiboas faber (Anura: Hylidae) during a Liophis miliaris (Serpentes: Colubridae) attack. Herpetology Notes 5: 187-188.

GIRAUDO, A. R., V. ARZAMENDIA \& P. CACCIALI, 2006. Geographic variation and taxonomic status of the southern most populations of Liophis miliaris (Linnaeus, 1758) (Serpentes: Colubridae). The Herpetological Journal 16(2): 213-220.

LIMA. A. C., 1940. Insetos do Brasil: hemípteros: 1-351. Escola Nacional de Agronomia, Rio de Janeiro.

LIMA, A. C., 2012. Revisão taxonômica da serpente neotropical Liophis miliaris (Linnaeus, 1758) (Serpentes: Colubridae): 1-109. Tese (Doutorado em Ciências Biológicas/Zoologia) - Museu Nacional/Universidade Federal do Rio de Janeiro, Rio de Janeiro.
LIMA, A. F. B. \& P. COLOMBO, 2008. Observação do comportamento predatório de Liophis miliaris orinus (Serpentes, Colubridae) em Hylodes meridionalis (Anura, Hylodidae), Serra Geral, Rio Grande do Sul, Brasil. Revista Brasileira de Zoociências 10(1): 73-76.

MARQUES, O. A. V. \& I. SAZIMA, 2004. História natural dos répteis da Estação Ecológica Jureia-Itatins. In: O. A. V. MARQUES \& W. DULEPA (Ed.): Estação Ecológica Jureia-Itatins: ambientes físico, flora e fauna: 257-277. Holos, Ribeirão Preto.

MARTINS, M., I. SAZIMA \& S. G. EGLER, 1993. Predators of the nest building gladiator frog, Hyla faber, in southeastern Brasil. Amphibia-Reptilia 14(3): 307-309. DOI: <http://dx.doi. org/10.1163/156853893X00507>.

MICHAUD, E. J. \& J. A. DIXON, 1989. Prey items of 20 species of the Neotropical colubrid snake genus Liophis. Herpetological Review 20: 39-41.

MOYA, G. M. \& F. MAFFEI, 2012. Predation on Dendropsophus elianeae (Napoli \& Caramaschi, 2000) (Anura: Hylidae) by Thamnodynastes hypoconia (Cope, 1860) (Squamata: Colubridae). Herpetology Notes 5: 261-262.

SAZIMA, I. \& M. MARTINS, 1990. Presas grandes e serpentes jovens; quando os olhos são maiores que a boca. Memórias do Instituto Butantan 52(3): 73-39.

SAZIMA, I. \& C. F. B. HADDAD, 1992. Répteis da Serra do Japi: notas sobre história natural. In: L. P. C. MORELLATO (Ed.): História natural da Serra do Japi: ecologia e preservação de uma área florestal no Sudeste do Brasil: 212-237. Editora da UNICAMP/ FAPESP, Campinas.

TOFT, C. A., 1985. Resource partitioning in amphibians and reptiles. Copeia 1985(1): 1-21.

TOLEDO, L. F., R. S. RIBEIRO \& C. F. HADDAD, 2007. Anurans as prey: an exploratory analysis and size relationships between predators and their prey. Journal of Zoology 271(2): 170-177. DOI: $<$ https://doi.org/10.1111/j.1469-7998.2006.00195.x>.

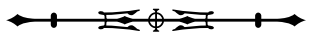

